\title{
Statistical Comparison and Improvement of Methods for Combining Random and Harmonic Loads
}

\author{
Andrew M. Brown" and David S. McGhee ${ }^{+}$ \\ NASA Marshall Space Flight Center, Marshall Space Flight Center, Alabama 35812
}

\begin{abstract}
Structures in many environments experience both random and harmonic excitation. $A$ variety of closed-form techniques has been used in the aerospace industry to combine the loads resulting from the two sources. The resulting combined loads are then used to design for both yield/ultimate strength and high-cycle fatigue capability. This paper examines the cumulative distribution function (CDF) percentiles obtained using each method by integrating the joint probability density function of the sine and random components. A new Microsoft ${ }^{\otimes}$ Excel spreadsheet macro that links with the software program Mathematica ${ }^{\otimes}$ is then used to calculate the combined value corresponding to any desired percentile along with a curve fit to this value. Another Excel macro is used to calculate the combination using a Monte Carlo simulation. Unlike the traditional techniques, these methods quantify the calculated load value with a consistent percentile. Using either of the presented methods can be extremely valuable in probabilistic design, which requires a statistical characterization of the loading. Also, since the CDF at high probability levels is very flat, the design value is extremely sensitive to the predetermined percentile; therefore, applying the new techniques can lower the design loading substantially without losing any of the identified structural reliability.
\end{abstract}

\section{Introduction}

$\mathrm{M}$ any structural components in dynamic environments experience both sinusoidal and random loadings at the same time. One example of such a structure is a component of a rocket engine. There are several sources that produce the random component of the excitation, including the gas generator and main combustion chamber. Simultaneously, the turbomachinery in the engine generates large harmonic loads due to the unavoidable unbalance in their rotors. In general, the structural dynamic response analyses for the random and harmonic cases are performed separately, and the results are then combined to obtain a design load. These loads are used to determine a level of alternating stress in the component for design to both ultimate/yield strength and for high-cycle fatigue (HCF). The standard industrial practice has been to use one of several closed-form equations for combining these two loads, and the selection of the proper equation has been the subject of some dispute. In an effort to generate a combined load consistent with the statistical characterization of the random component, the first author implemented the Monte Carlo technique for calculating the load as detailed in a previous paper. ${ }^{1}$ The paper presented here

\footnotetext{
ED21/Structural Dynamics \& Loads Group, and AIAA Member.

${ }^{+}$ED21/Structural Dynamics \& Loads Group.
} 
describes a more exact and easily implemented method for performing the calculation using a Microsoft ${ }^{\circledR}$ Excel macro to run a numerical integration technique in the software code Mathematica ${ }^{\circledR}$. This study also evaluates the closed-form methodologies used in industry and presents accurate closed-form curve fits of commonly used cumulative distribution function (CDF) percentiles for easy implementation in a production loads process.

The closed-form methods presently used in industry are all based upon the method used for obtaining design loads for purely random environments. Although there have been studies suggesting that the extreme-value distribution should be used for limit load design, ${ }^{2}$ a widely accepted practice is to use three times the root mean square (RMS) of the random load, $\sigma_{\text {ran }}$, for the design load, where the distribution is assumed to be Gaussian. This value will exceed the response $99.865 \%$ of the time. It has also been suggested that a value $<99.865 \%$ is acceptable for HCF design, such as $97.725 \%$, which is the percentile for 2 times the random RMS.

The most frequently used method for combining the two dynamic load components, referred to here as the standard method, simply uses the sum of the amplitude of the sine load, $A_{\sin }$, and $3 \times \sigma_{\text {ran }}{ }^{3}$

$$
\text { design load }=A_{\text {sin }}+3 \sigma_{\text {ran }}
$$

This technique assumes the sine peak value always occurs simultaneously with the peak random value. Another frequently applied method is to multiply 3 by the square root of the sums of mean square values of both the sines and the random, called the $3 \times$ ssMS method. ${ }^{4}$

$$
\text { design load }=3 \sqrt{\left(\sigma_{\sin }\right)^{2}+\left(\sigma_{\text {ran }}\right)^{2}}
$$

where $\sigma_{\text {sin }}$, the RMS of the sine wave, is equal to the sine amplitude divided by $\sqrt{2}$. This method treats the sine as if it had a normal probability distribution, which has a much larger range than a sine function. The $3 \times s s M S$ approach is similar to equations written to combine loads on launch vehicles, which do not have a substantial harmonic component but do have random loads from several sources. ${ }^{5}$ A third approach, which is proposed by Steinberg, ${ }^{6}$ is to take the root sum square of the peak values (called the peak method).

$$
\text { design load }=\sqrt{\left(A_{\mathrm{sin}}\right)^{2}+\left(3 \sigma_{\mathrm{ran}}\right)^{2}}
$$

Although it is stated in Ref. 6 that the standard method is too conservative and that it is convenient to use the suggested peak method, no mathematically rigorous justification is presented for using it for either ultimate/yield strength or HCF design. Finally, a major drawback of all of these closed-form methods is that none of them produce a design load that can be consistently associated with a particular probability level.

The integration technique described in this paper has been previously used by Schock, ${ }^{7}$ who was able to analytically calculate the joint cumulative density function for a single random load standard deviation of 1 and was able to generate curves of different CDF values corresponding to this value. Ferebee ${ }^{8}$ added to this work; however, the results do not appear to be consistent with the work presented below, and the methodology for performing the integration is not discussed.

\section{Loads Combination Using Probability Density Functions}

Since a sine wave considered by itself is not a random signal, it has always been unclear how to combine harmonic analysis results with output from a random excitation analysis to obtain a design load. The harmonic signal can, however, be defined as a stationary random process when combined with an independent Gaussian random signal since its phase relationship, $\phi$, with the random signal is random. ${ }^{9}$ If the harmonic signal is defined as 


$$
y_{\sin }=A(\omega t+\phi)
$$

where $\omega$ is the frequency of the signal and $A$ is the amplitude, then the argument $(\omega t+\phi)$ is therefore a random variable with a uniform distribution over the range $-\pi / 2$ to $\pi / 2$. The probability density function (PDF) of the sine function of this distribution is

$$
f(y)=\frac{1}{\pi A \sqrt{1-\left(\frac{y}{A}\right)^{2}}},
$$

shown graphically in Fig. $1 .^{10}$

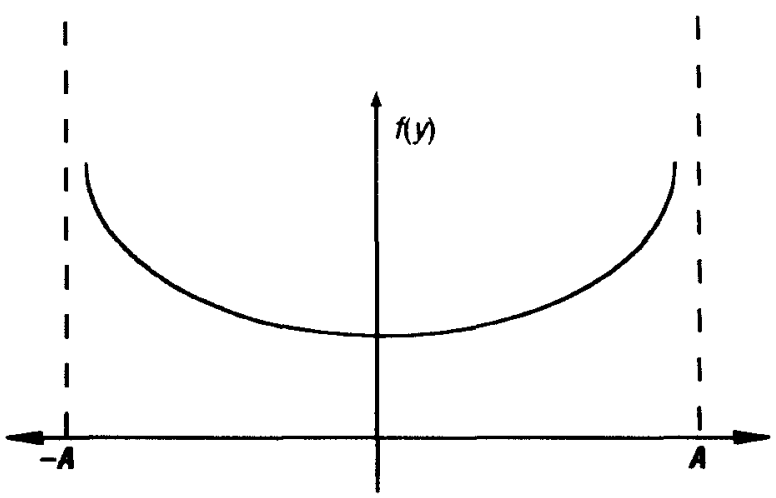

Figure 1: Probability density function $f(y)$ for the sine function.

The equivalent event technique can now be used to calculate the CDF, $F(z)$, of functions of random variables. For the function $z=x+y$, where $x$ and $y$ are random variables, the resultant $F(z)$ is defined in terms of the PDFs $f_{x}$ and $f_{y}$ as

$$
F(z)=\int_{-\infty}^{\infty}\left(\int_{-\infty}^{z-y} f_{x}(x) d x\right) f_{y}(y) d y
$$

If $x$ represents the random load, it can be characterized by a normal distribution with mean zero and standard deviation equal to the RMS from the random analysis. Substituting the normal PDF and the PDF shown in Eq. (5) into Eq. (6) results in

$$
F(z)=\int_{-A}^{A}\left(\frac{1}{\sigma_{\mathrm{ran}} \sqrt{2 \pi}} \int_{-\infty}^{z-y} \exp \left(-\left(\frac{x}{\sigma_{\mathrm{ran}}}\right)^{2}\right) d x\right) \frac{1}{\pi \sqrt{1-\left(\frac{y}{A}\right)^{2}}} d y
$$


where the integral over $\mathrm{y}$ is only evaluated from $-A_{\sin }$ to $A_{\sin }$ since the function is undefined outside of that range. A closed-form solution for this integral cannot be obtained; however, recent improvements in the software code Mathematica ${ }^{\circledR} 4.1$ have enabled it to be evaluated numerically for any value of $A, z$, and $\sigma_{\text {ran. The routine crashes if }}$ the integration is carried out over the range exactly from $-A$ to $A$, so the limits are instead set at $-A+\varepsilon$ to $A-\varepsilon$, where $\varepsilon$ is a very small value. The value of $\varepsilon$ was obtained by performing a series of runs with decreasing $\varepsilon$ until the solution converged; the final value obtained was 1 .1" $^{\prime \prime}$.

It is also of interest to obtain the solution for the inverse problem from that described above; i.e., what would the design load be that would yield a specific CDF value. In particular, if the $99.865 \%$ level has traditionally been chosen as an acceptable level of probability of excedance for designing to random loads, then it would be consistent to use that percentile to design for the combination of all dynamic loads. The Mathematica ${ }^{\circledR}$ Runge-Kutta FindRoot command can be implemented to calculate this load $z$ given the probability $F(z), A_{\text {sin }}$, and $\sigma_{\text {ran }}$.

To facilitate ease of use, both the inverse-integration technique and the Monte Carlo technique discussed in the author's previous paper are implemented as Microsoft ${ }^{\circledR}$ Visual Basic ${ }^{\circledR}$ macros launched from Excel. The listings are available in a NASA technical paper ${ }^{11}$ and in electronic format from the authors. In each case, any number of different combinations of random $1 \sigma$ values and sine amplitudes are listed in columns; these columns, along with an adjacent blank column, are chosen and the macro is selected and launched. The inverse-integration technique automatically writes the input values to a text file and launches the Mathematica ${ }^{B}$ kernel in the background that reads the data, performs the root search for the chosen probability level, and writes the result into a text file that is then read into the result column in Excel. This process takes $\approx 4.5 \mathrm{~s}$ for each combination on an Intel ${ }^{\circledast} \mathrm{Pentium}^{\circledR} 4$ $1.8 \mathrm{GHz}$ workstation with $500 \mathrm{MB}$ RAM running Microsoft ${ }^{\circledR}$ Windows 2000.

\section{Evaluation of Closed-Form Methods}

The integration method was used to evaluate the actual CDF values using each of the three closed-form methods. The sine and random inputs for this evaluation were chosen from the $\mathrm{MC}-1$ rocket engine developed at Marshall Space Flight Center, as well as for hypothetical cases with the ratio $x=\sigma_{\text {ran }} / A_{\text {sin }}$ ranging from 0.02 to 10 . A representative subset of the CDF results are shown in Table 1 . The combination load values are also compared with loads obtained for a $99.865 \%$ probability level using the inverse-integration method and the Monte Carlo method, with the inverse-integration method being chosen as the baseline. There are several methods available for choosing a sample size that will result in accurate answers for Monte Carlo; but for conservatism, a study was performed for this research that showed that load combinations converged using 400,000 samples (a value much higher than that obtained using the closed-form sample size determination methods). These Monte Carlo results are in very close agreement with the inverse-integration technique. As expected from their derivation, both the standard and the $3 \times$ ssMS methods always exceed the baseline value by varying amounts. The "peak" method always yields a value less than the baseline. The standard and 3xssMS results are shown in Fig. 2, which is a plot of the percentage overshoot over the baseline versus the ratio $x=\sigma_{\text {ran }} / A_{\text {sin }}$ for each method.

The functional relationships identified in figure 2 were examined for various values of the parameters and were seen to be only a function of the ratio described above. This independent functional relationship identified the possibility of creating a closed-form equation that would exactly match the values obtained using the inverseintegration method for a specific probability level. The Mathematica ${ }^{\circledR} 4.1$ curve-fit routine was used to examine different categories of functions for fitting the overshoot, resulting in Eq. (8), with the comparison of the function with the data shown in Fig. 3.

$$
\text { overshoot }=0.0323928 e^{-x}\left(-\frac{0.00257298}{x^{5}}+\frac{0.0722376}{x^{4}}-\frac{0.715841}{x^{3}}+\frac{2.64516}{x^{2}}+\frac{1.24289}{x}\right)
$$


Table 1: Comparison of all methods.

\begin{tabular}{|c|c|c|c|c|c|c|c|c|c|}
\hline $\begin{array}{c}\text { Sine } \\
\text { Amplitude }\end{array}$ & $\begin{array}{c}1 \sigma \\
\text { Random }\end{array}$ & $\begin{array}{l}\text { Random/ } \\
\text { Sine }\end{array}$ & $\begin{array}{l}\text { Load Using } \\
\text { Integration } \\
\text { Method for } \\
\mathbf{9 9 . 8 6 5 \%} \\
\text { (baseline) }\end{array}$ & $\begin{array}{c}\text { Load, Monte } \\
\text { Carlo Method } \\
\text { for } 99.86 \% \\
400,000 \\
\text { Samples }\end{array}$ & $\begin{array}{l}\text { Load, } \\
\text { Standard } \\
\text { Method } \\
\mathbf{A + 3 \sigma}\end{array}$ & 1 & $\begin{array}{c}\text { Overshoot } \\
\text { From } \\
\text { Baseline } \\
(\%)\end{array}$ & $\begin{array}{l}1 \\
1 \\
1\end{array}$ & $\begin{array}{l}\text { CDF Value } \\
\text { From } \\
\text { Integration } \\
(\%)\end{array}$ \\
\hline 10 & 5 & 0.5 & 22.034 & 22.031 & 25 & & 13.5 & & 99.970 \\
\hline 5 & 5 & 1 & 17.668 & 17.653 & 20 & 1 & 13.2 & I & 99.957 \\
\hline 5 & 20 & 4 & 60.915 & 60.888 & 65 & 1 & 6.7 & I & 99.919 \\
\hline 26 & 4 & 0.154 & 34.760 & 34.772 & 38 & I & 9.3 & 1 & 99.983 \\
\hline 97 & 14.67 & 1.151 & 129.081 & 129.195 & 141.01 & 1 & 9.2 & I & 99.986 \\
\hline 50 & 98.7 & 1.974 & 313.047 & 313.422 & 346.1 & 1 & 10.6 & I & 99.951 \\
\hline 64 & 109.33 & 1.708 & 352.240 & 353.079 & 391.99 & i & 11.3 & $i$ & 99.955 \\
\hline
\end{tabular}

\begin{tabular}{|c|c|c|c|c|c|c|c|c|c|c|}
\hline $\begin{array}{c}\text { Sine } \\
\text { Amplitude }\end{array}$ & $\begin{array}{c}1 \sigma \\
\text { Random }\end{array}$ & $\begin{array}{c}\text { Load, } \\
\text { 3×ssiMs }\end{array}$ & $\begin{array}{l}1 \\
1 \\
1 \\
1\end{array}$ & $\begin{array}{c}\text { Overshoot } \\
(\%)\end{array}$ & $\begin{array}{l}1 \\
1 \\
1 \\
1\end{array}$ & $\begin{array}{l}\text { CDF Value } \\
\text { From } \\
\text { Integration } \\
(\%)\end{array}$ & $\begin{array}{l}\text { Load, } \\
\text { Peak } \\
\text { Method } \\
\text { ss }(\sigma, A)\end{array}$ & I & $\begin{array}{c}\text { Overshoot } \\
(\%)\end{array}$ & $\begin{array}{c}\text { CDF Value } \\
\text { From } \\
\text { Integration } \\
(\%)\end{array}$ \\
\hline 10 & 5 & 25.981 & 1 & 17.9 & I & 99.990 & 18.028 & I & -18.2 & 93.930 \\
\hline 5 & 5 & 18.371 & 1 & 4.0 & 1 & 99.912 & 15.811 & I & -10.5 & 94.896 \\
\hline 5 & 20 & 60.930 & 1 & 0.03 & i & 99.865 & 60.208 & i & -1.2 & 95.758 \\
\hline 26 & 4 & 56.445 & 1 & 62.4 & 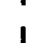 & 100.000 & 28.636 & 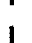 & -17.6 & 94.291 \\
\hline 97 & 14.67 & 210.422 & 1 & 63.0 & 1 & 100.000 & 106.517 & & -17.5 & 94.316 \\
\hline 50 & 98.7 & 314.524 & , & 0.5 & | & 99.871 & 300.292 & & -4.1 & 95.534 \\
\hline 64 & 109.33 & 354.978 & 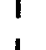 & 0.8 & & 99.875 & 334.176 & & -5.1 & 95.443 \\
\hline
\end{tabular}

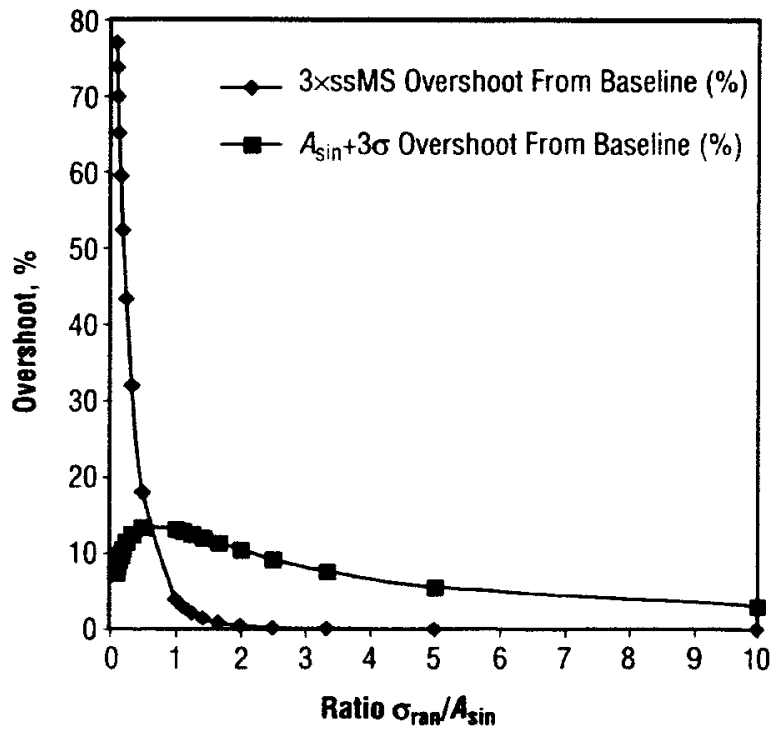

Figure 2: Percent overshoot from baseline using traditional methods.

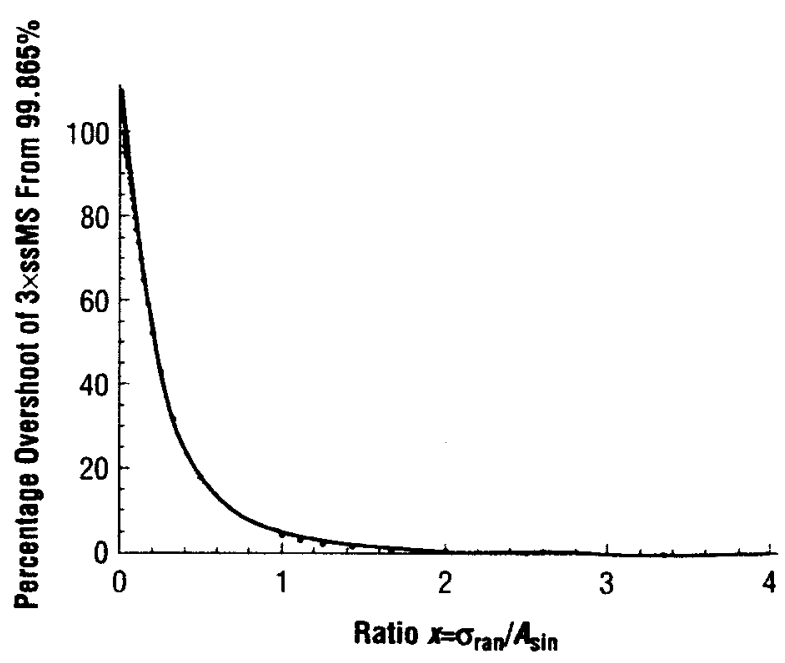

Figure 3: Curve fit of overshoot of 3xssMS method over CDF of $99.865 \%$. 
The desired closed-form equation is therefore

$$
\text { design load }=\frac{3 \sqrt{\frac{A_{\mathrm{sin}}^{2}}{\sqrt{2}}+\sigma_{\mathrm{ran}}^{2}}}{1+\text { overshoot }}
$$

A comparison of the baseline values with those obtained using this new closed-form solution shows the agreement to be excellent, with only one of the points showing an overshoot of $>1 \%$. This equation can be directly implemented into a spreadsheet for easy application in the production loads process. A similar equation has been generated that can be used to obtain a desired combined percentile of $97.725 \%$ for the design load, which, as noted earlier, has been suggested as a more appropriate value for $\mathrm{HCF}$ calculations.

$$
\operatorname{design~load~}_{97.725 \%}=\frac{2 \sqrt{\frac{A_{\text {sin }}^{2}}{\sqrt{2}}+\sigma_{\text {ran }}^{2}}}{1+\exp \left(-2.0115 x^{1.15}\right)\left(\frac{5.003 \times 10^{-7}}{x^{5}}-\frac{3.0689 \times 10^{-5}}{x^{4}}+\frac{9.304 \times 10^{-4}}{x^{3}}-\frac{0.01469}{x^{2}}+\frac{0.1177}{x}\right)}
$$

\section{Conclusion and Areas of Future Work}

The probability values associated with several widely used methods for combining harmonic and random loads have been determined. In addition, two new Excel macros have been written that calculate the combined load value for any specified probability level. Because of the speed of desktop computers, these macros can be executed extremely easily and quickly. Closed-form solutions approximating the probabilistic percentiles of $99.865 \%$ and $97.725 \%$ have also been determined. The results presented in this paper can assist the analyst in choosing a method for combining these loads; obtaining an accurate value can be critical for the design of weight-sensitive aerospace structures. One avenue of future work in this area is incorporating the effect of the correlation of the sine loads for harmonics of a single rotating shaft. Another will be to implement the methodology discussed here to the extreme value distribution, which may be more accurate for HCF calculations.

\section{References}

'Brown, A. M., "Combining Loads From Random and Harmonic Excitation Using the Monte Carlo Technique," Journal of Spacecraft and Rockets, Vol. 37, No. 4, July-August 2000, pp. 541-543.

${ }^{2}$ Merchant, D. H., "Methods for Combining Payload Parameter Variations with Input Environment," NASA CR-149939, 1976.

${ }^{3}$ Rocketdyne Structural Dynamics Manual, Rocketdyne Corporation, Vol. 2, Sec. 2.2-6, 1989.

${ }^{4}$ Wen, Y.-K., Structural Load Modeling and Combination for Performance and Safety Evaluation, Elsevier, Amsterdam, 1990 , p. 211.

${ }^{5}$ Macheske, V., Womack J., and Binkley, J., "A Statistical Technique for Combining Launch Vehicle Atmospheric Flight Loads," AIAA Paper No. 93-0755, 1993.

${ }^{6}$ Steinberg, D. S., Vibration Analysis for Electronic Equipment, John Wiley \& Sons, New York, 1988, p. 249.

${ }^{7}$ Schock, R., and Tuell, L., "An Investigation into the Probabilistic Combination of Quasi-Static and Random Accelerations," AIAA Paper No. 84-0908, 1984.

${ }^{8}$ Ferebee, R., "Loads Combination Research at Marshall Space Flight Center," NASA/TM-2000-210331, 2000.

${ }^{9}$ Wirshing, P., Paez, T., and Ortiz, K., Random Vibrations: Theory and Practice, John Wiley \& Sons, New York, 1995 , p. 81.

${ }^{10}$ Rheinfurth, M., and Howell, L. W., "Probability and Statistics in Aerospace Engineering," NASA TP 1998-207194, 1998.

${ }^{11}$ Brown, A. M., and McGhee, D. S., "Statistical Evaluation and Improvement of Methods for Combining Random and Harmonic Loads," NASATP-2003-212257, 2003. 